\title{
DELTA WING WITH BLUNTED LEADING EDGES AT ANGLE OF ATTACK IN HYPERSONIC FLOW
}

\author{
P. Vashchenkov, A. Kashkovsky, and M. Ivanov \\ Institute of Theoretical and Applied Mechanics \\ Siberian Branch of the Russian Academy of Sciences \\ 4/1 Institutskaya Str., Novosibirsk 630090, Russia
}

\begin{abstract}
A hypersonic rarefied diatomic gas flow around a delta wing with a blunted leading edge at an angle of attack of $10^{\circ}$ is considered. Flow fields on the leeward and windward sides are obtained. Specific features of surface distributions of thermal characteristics on the side edge are studied.
\end{abstract}

\section{INTRODUCTION}

In designing reentry vehicles with a high lift-to-drag ratio, the lifting surface is usually a delta wing. To ensure acceptable values of the temperature of the vehicle structure, the side edges and the wing tip are made with a certain bluntness radius depending on the thermal resistance of the material used. The air flow in the vicinity of such a wing, especially at an angle of attack, has a complicated three-dimensional structure. Experimental studies of aerothermodynamic loading of such vehicles show that the flow at an angle of attack involves the formation of vortices, which induce elevated heat fluxes on the leeward side (see, for example, [1-3]). All these studies were performed under continuum conditions at Reynolds numbers $\operatorname{Re}>10^{5}$. To obtain a comprehensive estimate of the delta wing parameters in all ranges of free-stream parameters, it is necessary to know the behavior of the flow around the wing in a low-density gas flow. The present paper describes the flow around a delta wing with a blunted leading edge at an angle of attack of $10^{\circ}$ and Reynolds numbers $\operatorname{Re}<10^{5}$.

There were two goals in our calculations: to study the process of vortex formation on the leeward side of the delta wing and to consider specific features of the flow around the leading edge blunted with a small radius. A particular attention was paid to heat fluxes with allowance for the temperature nonequilibrium. 


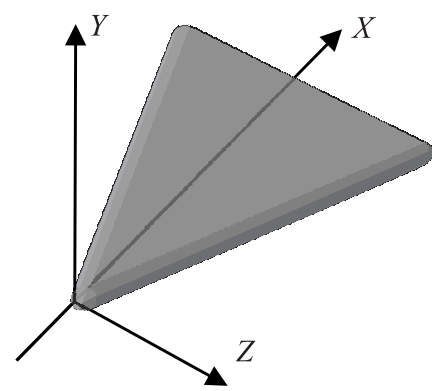

Figure 1 Model geometry

A diatomic gas flow with Reynolds numbers $\operatorname{Re} \sim 10^{4}$ was numerically simulated. The computations were performed at angles of attack equal to $0^{\circ}$ and $10^{\circ}$. Flow fields and surface distributions of aerothermodynamic parameters were obtained.

\section{MODEL GEOMETRY}

The model geometry is a delta wing with a sweep angle of $75^{\circ}$. The wing length is $12 \mathrm{~cm}$, the bluntness radius of the side edges is $3 \mathrm{~mm}$, and the wing tip and rear corners are spherically blunted with a radius of $3 \mathrm{~mm}$ (Fig. 1).

\section{FLOW PARAMETERS}

A diatomic gas flow around a delta wing was studied. The flow parameters were the Mach number $\mathrm{M}=10$ and the Reynolds number $\operatorname{Re}=2440,1220$, 12,200, 24,400, and 61,000. The wall temperature was assumed to be constant: $T_{w} / T_{0}=0.4$. The stagnation temperature was $T_{0}=750 \mathrm{~K}$.

\section{NUMERICAL METHOD}

Simulations were performed with the Direct Simulation Monte Carlo (DSMC) method. In this method, the gas is considered as a set of a finite number of particles that move in the flow and collide with each other and with the body. Intermolecular collisions were calculated on the basis of a random law with allowance for the internal energy. As the DSMC method allows the internal energy to be taken into account, it can be used to model nonequilibrium processes that occur, for instance, in shock waves.

The SMILE software system [4] was used for computations. The internal energy relaxation was obtained with the Larsen-Borgnakke model. The number of collisions was determined by the majorant frequency method. Up to 256 processors of the computational cluster of the Interdepartmental Supercomputer Center (Moscow), Siberian Supercomputer Center (Novosibirsk), and Khristianovich Institute of Theoretical and Applied Mechanics of the Siberian Branch of the Russian Academy of Sciences were used in computations. The total time required for computations was approximately 7000 processor-hours. 


\section{RESULTS}

Figure $2 a$ shows the temperature fields around the wing and the surface distributions of the heat-transfer coefficient $C_{h}=Q /\left(\left(\rho V^{3} / 2\right) S\right)$ obtained in computations for the most rarefied flow $(\operatorname{Re}=244)$. It is seen that the heat-transfer coefficient on the leeward side of the wing in the most rarefied flow remains almost constant. In less rarefied flows $(\operatorname{Re}=12,200$ and $R e=61,000)$, the heattransfer coefficient on the leeward side monotonically decreases with the distance from the wing edge (Figs. $2 b$ and $2 c$ ). There are no specific features that would testify the presence of separation regions or elevated heating zones on the leeward side in all cases considered.

To estimate the possibility of separation on the leeward side of the wing, let consider the distribution of the friction coefficient along the $Z$ axis aligned across the flow, along the surface from the plane of symmetry of the wing to the wing edge. If the flow becomes separated, the condition $C_{f, z}>0$ is satisfied, i. e., the flow is directed from the middle of the wing to its edge. The distributions $C_{f, z}(z)$ along the trailing edge of the wing for three Reynolds numbers considered are shown in Fig. 3. The behavior of $C_{f, z}(z)$ remains almost unchanged along the wing in the most rarefied flow $(\operatorname{Re}=244)$; in the cases with $\operatorname{Re}=12,200$ and $\operatorname{Re}=61,000$, the value of $C_{f, z}(z)$ increases as the wing edge is approached, but the sign is not changed. It is only on the side surface that $C_{f, z}(z)$ becomes positive, which testifies that the streamlines in this area are aligned along the wing edge.

Figure 4 shows the distributions of translational and rotational temperatures in the flow near the lower surface of the wing at the Reynolds number $\operatorname{Re}=61,000$. The left and right boundaries of the plots correspond to the freestream parameters and to the flow in the aerodynamic wake behind the wing, respectively. As the stagnation temperature is rather low $(750 \mathrm{~K})$, it is clear that there is practically no excitation of the vibrational mode. For this reason, it is not shown in these graphs. The translational temperature reaches higher values in the shock wave than the rotational temperature. When the wing edge is approached, relaxation occurs in the flow, and the translational and rotational temperatures level off. Another nonequilibrium zone is formed in the wake behind the wing where the translational temperature decreases faster than the rotational temperature.

Figure 5 shows the distribution of the heat-transfer coefficient along the side edge of the wing from the model tip $(S=0)$ to the end of the side edge at three Reynolds numbers. The distribution obtained at $\mathrm{Re}=61,000$ almost coincides with the distribution at $\mathrm{Re}=12,200$. There is a dip in the heat-transfer coefficient curve at the model tip, because the point where the maximum value is obtained for the model at an angle of attack is located below the axis of symmetry. 


\section{$T / \mathrm{K}$}

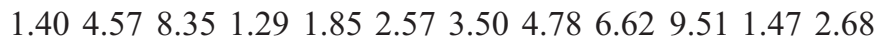

$\begin{array}{llllllllllll}-4.5 & 8.0 & 2.2 & 4.1 & 6.3 & 9.1 & 1.2 & 1.7 & 2.5 & 3.6 & 5.7 & 1.0 \\ C_{h} & & & & & & & & \end{array}$
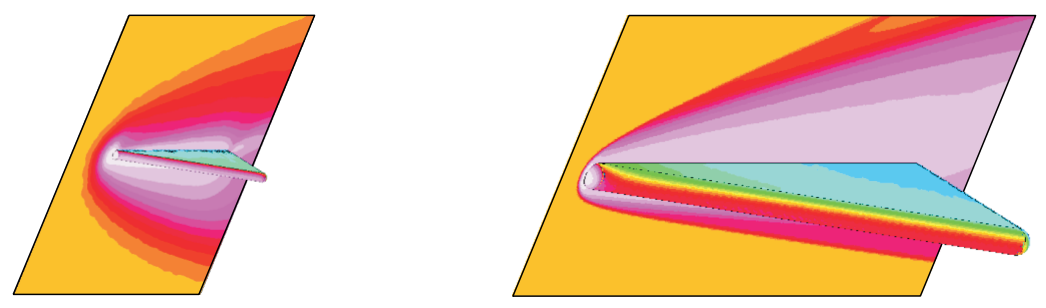

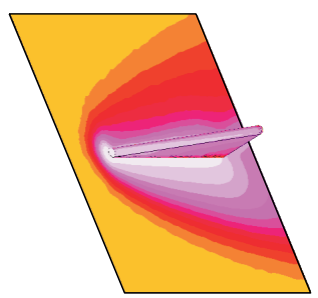

(a)

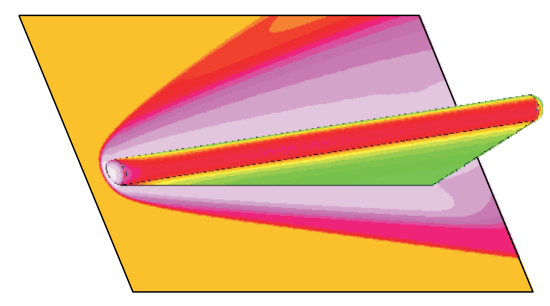

(b)
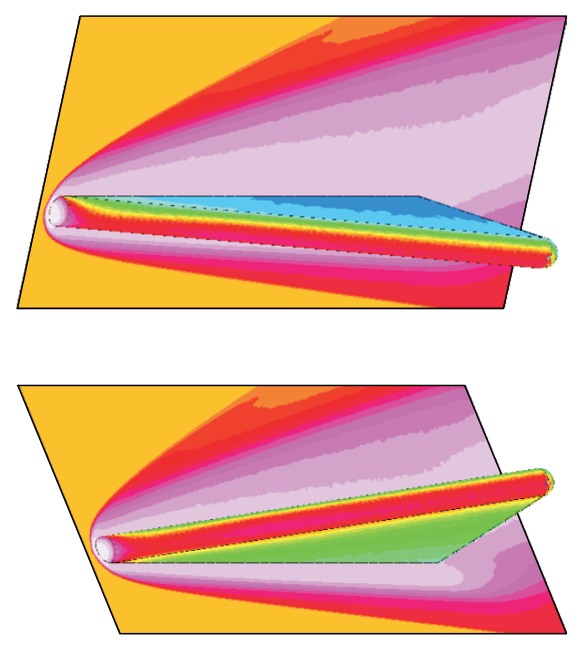

(c)

Figure 2 Temperature field and surface distribution of the heat-transfer coefficient: (a) $\operatorname{Re}=244 ;(b)$ 12,200; and (c) $\operatorname{Re}=61,000$. 


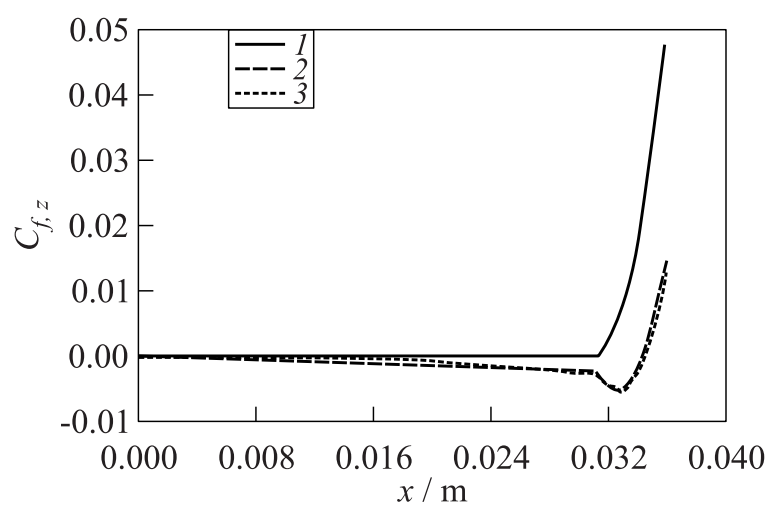

Figure 3 Distribution of the $z$ component of the friction coefficient near the trailing edge of the wing along the $Z$ axis: $1-\operatorname{Re}=244 ; 2-12,200$; and $3-\operatorname{Re}=61,000$

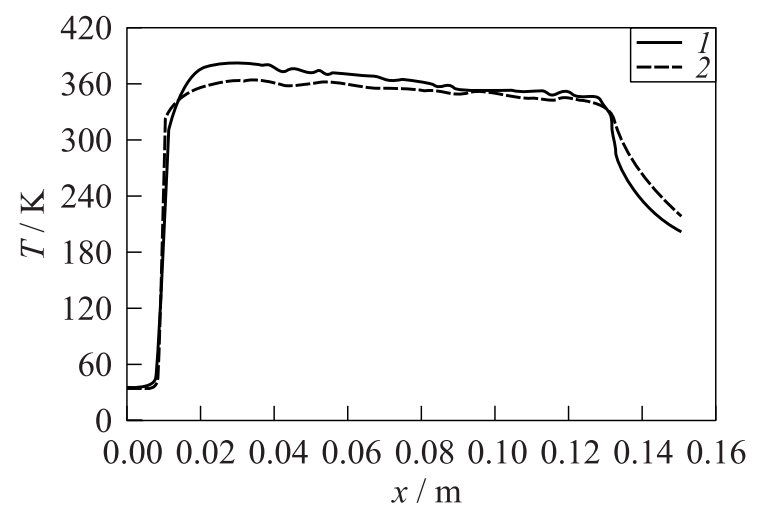

Figure 4 Distributions of translational (1) and rotational (2) temperatures in the flow near the lower surface of the wing

Another interesting fact is the nonmonotonic behavior of the curve $C_{h}(S)$ in the dense flow $(\operatorname{Re}>12,200)$. In the rarefied flow, $C_{h}$ monotonically decreases along the entire side edge. Its behavior is illustrated in more detail in Fig. 6, which shows the curves for the cases with $\operatorname{Re}=61,000$ and $R e=12,200$ only. It is seen that the value of $C_{h}$ on the side edge increases approximately by $20 \%$ in the range of $S$ from 1.7 to $5 \mathrm{~cm}$ and then monotonically decreases.

The dependence $C_{h}(S)$ behaves in this manner only if the model is aligned at an angle of attack. At the zero angle of attack, the heat-transfer coefficient reaches the maximum value at the model tip; then it decreases and remains 


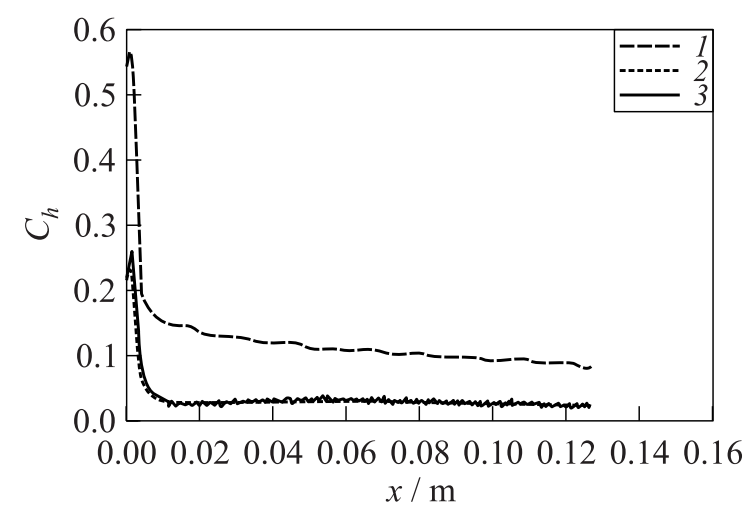

Figure 5 Distribution of the heat-transfer coefficient on the side edge of the wing: $1-\operatorname{Re}=244 ; 2-12,200 ;$ and $3-\operatorname{Re}=61,000$

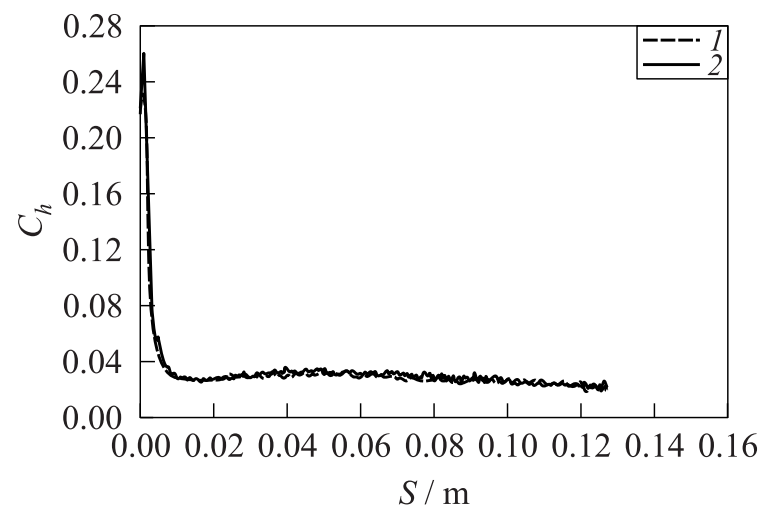

Figure 6 Distributions of the heat-transfer coefficient on the side edge of the wing at $\operatorname{Re}=12,200(1)$ and $61,000(2)$ (angle of attack $=10^{\circ}$ )

almost constant along the entire side edge. This can be seen in Fig. 7, which shows the distribution $C_{h}(S)$ obtained in computations with $\operatorname{Re}=61,000$ at the zero angle of attack. For comparison, Fig. 7 also shows the computed curve with the same Reynolds number at the angle of attack equal to $10^{\circ}$. The local value of $C_{h}$ at the initial segment of the side surface at a small angle of attack $\left(10^{\circ}\right)$ remains almost unchanged.

Figure 8 shows the number density fields obtained in computations at Re $=61,000$ for angles of attack equal to $0^{\circ}$ and $10^{\circ}$. The computation for the zero angle of attack was performed for the upper half of the wing with allowance for the axis of symmetry. 


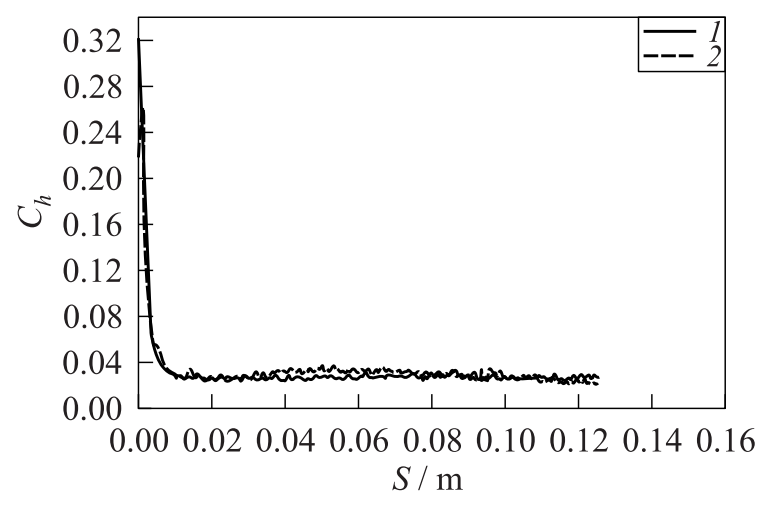

Figure 7 Distribution of the heat-transfer coefficient on the side edge of the wing at $\operatorname{Re}=61,000: 1-$ angle of attack $=0^{\circ}$; and $2-$ angle of attack $=10^{\circ}$
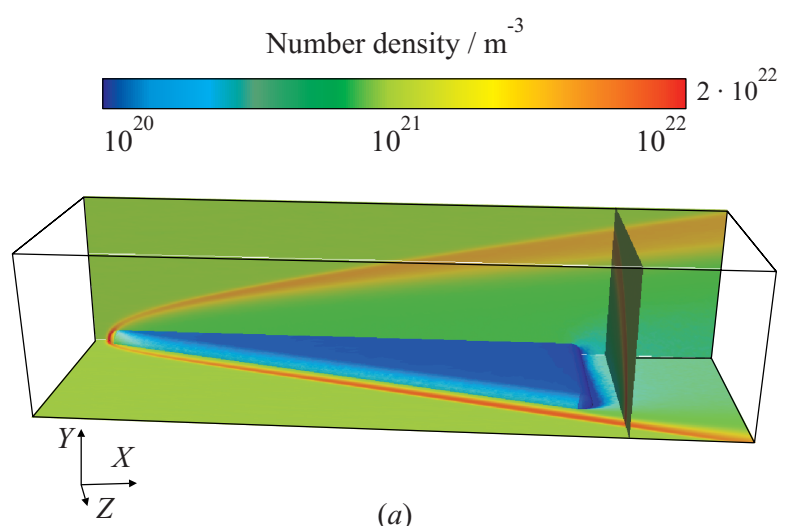

(a)

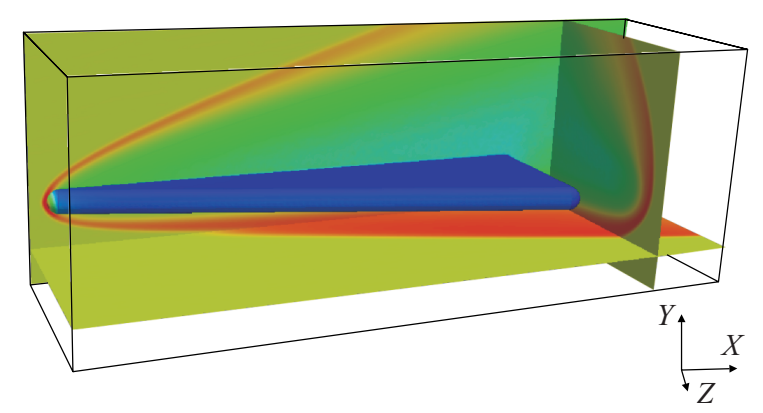

(b)

Figure 8 Number density flowfield distribution at $\operatorname{Re}=61,000:(a)$ angle of attack $=0^{\circ}$; and $(b)$ angle of attack $=10^{\circ}$. 


\section{CONCLUDING REMARKS}

The aerodynamic characteristics of a delta wing at an angle of attack in a hypersonic flow with the Reynolds number ranging from 244 to 62,000 are calculated. The results obtained show that the flow is laminar in the cases considered, and no separation regions observed in the continuum regime are formed here.

Despite the low stagnation temperature, there is rotational-translational nonequilibrium in the flow, which should be taken into account in analyzing heat fluxes.

For convenience of presentation and analysis, the results obtained will be incorporated into an aerodynamic database developed within the framework of Grant No. 09-07-00480 of the Russian Foundation for Basic Research.

\section{ACKNOWLEDGMENTS}

This work was supported by the Russian Foundation for Basic Research (Grant Nos. 10-08-01203, 11-01-91162, and 11-07-00135), by the Lavrent'ev Grant for young researchers entitled "High-altitude aerodynamics of promising space vehicles with allowance for nonequilibrium chemical reactions," and Russia-Taiwan Research Cooperation Project "Numerical Study of Rarefaction Effects and Thermochemical Nonequilibrium Problems on Hypersonic Flow around Space Vehicles."

\section{REFERENCES}

1. Stanbrook, A., and L. C. Squire. 1964. Possible types of flow at swept leading edges. Aeronaut. Quart. 15(2):72-82.

2. Brodetsky, M. D., and A. M. Shevchenko. 1990. Some features of a separated flow and supersonic vortex structure at the leeside of a delta wing. Separated Flows and Jets: IUTAM Symposium Proceedings. Novosibirsk. 341-44.

3. Brodetsky, M. D., A. M. Kharitonov, E. Krause, A. A. Pavlov, S. B. Nikiforov, and A. M. Shevchenko. 2000. Experimental investigation of the supersonic flow on the lee side of a delta wing: Experimental techniques and results. ICMAR 2000 Proceedings. Russia.

4. Ivanov, M. S., G. N. Markelov, and S. F. Gimelshein. 1998. Statistical simulation of reactive rarefied flows: Numerical approach and applications. 7th Joint Thermophysics and Heat Transfer Conference Proceedings. 